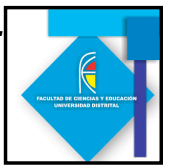

\title{
EXPERIMENTOS DEMOSTRATIVOS PARA LA CONSTRUCCIÓN DE CONCEPTOS EN TORNO A LAS ONDAS SONORAS
}

\section{DEMONSTRATION EXPERIMENTS FOR THE CONSTRUTION OF CONCEPTS ABOUT THE SOUNDS WAVES}

\author{
Angelica Gómez ${ }^{1}$ \\ Montes C. M. ${ }^{2}$
}

\begin{abstract}
Resumen
El presente trabajo surge de un proceso de investigación en el aula y abarca el diseño, elaboración e implementación de una estrategia de aula basada en el uso de experimentos demostrativos, para la construcción de conceptos en torno a las ondas sonoras.

La estrategia de aula tiene como fundamento pedagógico el constructivismo liderado por Novak, David Ausubel y Gowin centrándose en la propuesta de aprendizaje significativo de Ausubel. Se utiliza el experimento demostrativo y un módulo de trabajo diseñado, como materiales potencialmente significativos para la construcción del conocimiento y se orienta al estudiante hacia una actitud de aprendizaje significativa a través de preguntas insertadas.
\end{abstract}

Se realiza la implementación del trabajo en el aula del grado Noveno del colegio Nuestra Señora de la Salette, para finalmente analizar la incidencia que tiene la estrategia de aula en el aprendizaje de los estudiantes en torno a las ondas sonoras.

Palabras Clave: Experimentos demostrativos, aprendizaje significativo, ondas sonoras, grado noveno, módulo de trabajo.

\begin{abstract}
This work stems from a research process in the classroom and covers the design, development and implementation of a classroom strategy based on demonstration experiments, for the construction of concepts about sound waves.

Classroom strategy that relies on constructivist teaching led by Novak, Gowin and David Ausubel, the focus is the significant learning proposal by Ausubel. Demonstrative experiments and the working module are used as a potentially significant material for the
\end{abstract}

\footnotetext{
${ }^{1}$ Licenciada en Física, Universidad Pedagógica Nacional, angelicamgt@hotmail.com

${ }^{2}$ Docente, Departamento de Física - Universidad Pedagógica Nacional., cmontes@pedagogica.edu.co
} 


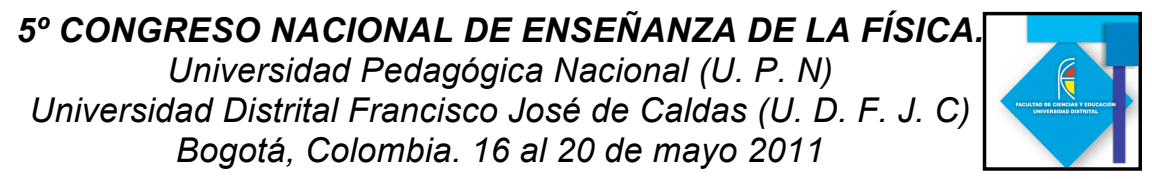

construction of knowledge. Perhaps the teacher guides the student to bilding his learning through inserted questions.

The implementation of the work realizes in the ninth grade of the school Nuestra Señora de la Salette, and finally analyzes the impact that has a classroom strategy in the learning of the students around the sounds waves.

Keywords: demonstration experiments, significant learning, sound waves, ninth grade, working module.

\section{Contextualización}

Inicialmente se hace una contextualización del trabajo desde la práctica y la teoría, la contextualización desde la práctica inicia con una descripción de la población a la que se dirige la estrategia de aula, para establecer la situación problemática que enmarca la pregunta de investigación, la alternativa de solución y la justificación.

Luego de una observación detallada en el aula de grado noveno del Colegio Nuestra Señora de la Salette, se identifica que no se llevan a cabo experimentos en la clase de física, razón por la cual se plantea la pregunta de investigación ¿Qué incidencia tiene una estrategia de aula basada en el uso de experimentos demostrativos, en el estudio que los estudiantes de grado Noveno hacen de las características ondulatorias del sonido? A propósito de que el sonido es el tema a trabajar en el segundo semestre académico del año y que el colegio no cuenta con un laboratorio para llevar a cabo los experimentos.

Se diseña la estrategia de aula teniendo como fundamento pedagógico el constructivismo liderado por Novak, Ausubel y Gowin, tomando como base la propuesta de aprendizaje significativo de Ausubel. Se utiliza el experimento demostrativo y un módulo de trabajo diseñado, como materiales potencialmente significativos para la construcción del conocimiento y se orienta al estudiante hacia una actitud de aprendizaje significativa a través de preguntas insertadas.

\section{Estructura de la estrategia de aula}

Inicialmente se realiza una organización jerárquica de los conceptos que se desean abordar, tal como lo plantea el constructivismo.

Se considera que en el primer lugar de jerarquía se encuentra reconocer el sonido como una onda, pues esa concordancia le permite al estudiante empezar a establecer relaciones entre las características que ya conoce de las ondas y la nueva onda que está estudiando.

Una vez el estudiante reconoce el sonido como una onda, debe caracterizarlo según su medio de propagación y según su dirección de propagación, aplicando lo que conoce previamente. Esa relación le permite evidenciar desde la naturaleza el concepto de onda que maneja y la forma en que las clasifica. 


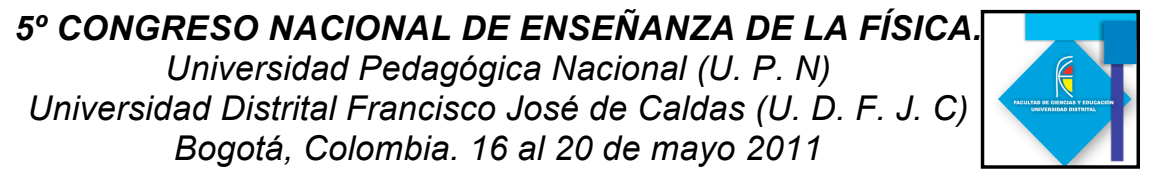

Luego será necesario que el estudiante identifique la frecuencia de la onda sonora como el tono del sonido, la amplitud de la onda como el volumen del sonido y el timbre como una misma frecuencia emitida por fuentes diferentes, para finalizar haciendo una relación entre la frecuencia, la longitud de onda y la velocidad del sonido a través del análisis del efecto Doppler.

La construcción conceptual será acompañada por el maestro, quien además de proporcionar al estudiante las herramientas para que descubra por sí mismo las relaciones que pueden establecerse, debe orientarlo constantemente en su construcción de conocimiento por medio de preguntas orientadoras.

Finalmente se realizará una discusión alrededor de las características ondulatorias de los géneros musicales favoritos de los estudiantes.

Para el trabajo en clase y como herramienta de recolección de la información, se diseña un modulo de trabajo, organizado en cuatro unidades (Tabla 1), que contiene las preguntas orientadoras más generales de cada una de las experiencias a tratar.

\begin{tabular}{|l|l|l|}
\hline No & Nombre & Objetivo de la unidad \\
\hline 1. & $\begin{array}{l}\text { Exploremos lo que } \\
\text { conoces del tema... }\end{array}$ & $\begin{array}{l}\text { Introducir a los estudiantes al estudio del sonido y determinar el nivel } \\
\text { de construcción conceptual que tienen en torno a él. }\end{array}$ \\
\hline 2. & ¿Qué es el sonido? & Identificar el sonido como una onda mecánica y longitudinal. \\
\hline 3. & $\begin{array}{l}\text { Analicemos las } \\
\text { características del } \\
\text { sonido. }\end{array}$ & $\begin{array}{l}\text { Relacionar la frecuencia de la onda sonora con el tono del sonido, la } \\
\text { amplitud de la onda sonora con el volumen del sonido y la fuente de } \\
\text { emisión con el timbre del sonido. }\end{array}$ \\
\hline 4. & Efecto Doppler & $\begin{array}{l}\text { Conocer la relación entre longitud de onda, frecuencia, velocidad de } \\
\text { propagación y velocidad de movimiento de una fuente sonora. }\end{array}$ \\
\hline
\end{tabular}

Tabla 1. Unidades y objetivos de la estrategia de aula

\section{Material de trabajo}

Se diseña y elabora un módulo de acústica que es distribuido a todos los estudiantes (figura 1)
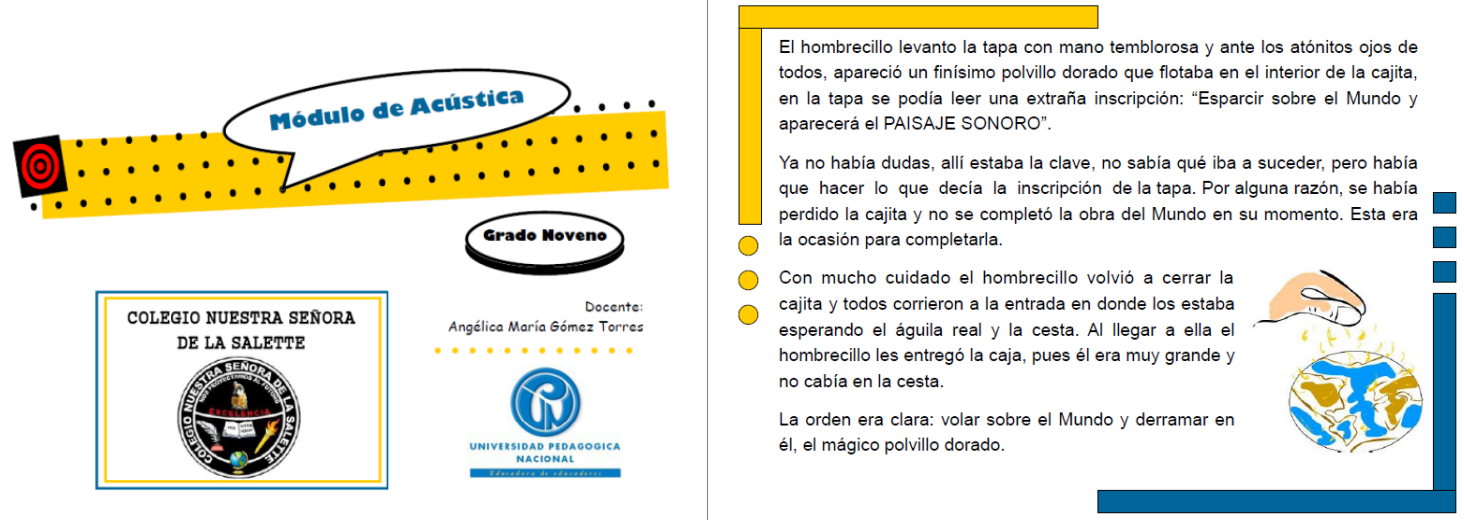

Imagen 1. Módulo de trabajo

y los experimentos demostrativos (figura 2) 

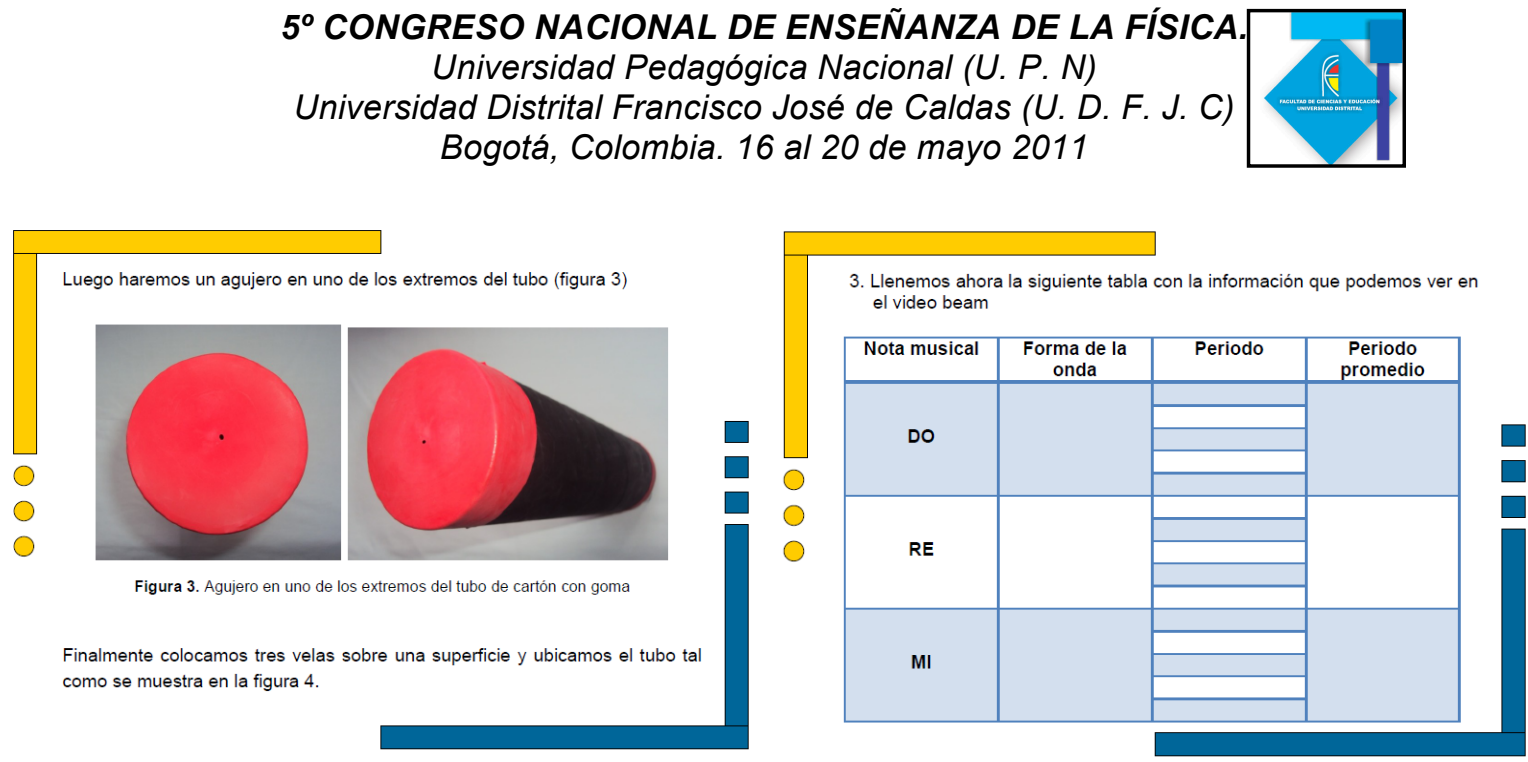

Figura 2. Experimentos demostrativos

Paralelo a esta elaboración de diseña la estrategia de aula y las técnicas de recolección de información, se elaboran circulares para los padres de familia en donde autorizan el registro de video de los estudiantes. Con el material diseñado se realiza la incursión en el aula. Finalmente se analiza la implementación en el aula a la luz de los objetivos planteados en la estrategia de aula, y se determina la incidencia de la misma en el estudio que los estudiantes de grado Noveno hacen de las características ondulatorias del sonido.

\section{Conclusiones}

Se encuentran orientadas en cuanto al uso de experimentos demostrativos, al uso de un módulo de trabajo, a la orientación del trabajo por medio de preguntas y a la estrategia de aula en general.

\section{En cuanto al uso de experimentos demostrativos}

Utilizar experimentos demostrativos permite a los estudiantes observar el fenómeno tan cercano a la vida cotidiana que la temática adquiere mayor grado de importancia para ellos.

Manejar un solo montaje experimental para todo un curso con pocos estudiantes, le permite al maestro un mejor control de grupo, además de un control sobre el material de laboratorio cuidando que no sea mal utilizado.

\section{En cuanto al uso de un módulo de trabajo}

Manejar un módulo de trabajo le permite al estudiante y al maestro tener un control sobre la secuencia de la clase y mantener clara la meta de aprendizaje.

Los estudiantes perciben un mayor interés del maestro hacia ellos, lo que los motiva a participar activamente en clase.

El módulo de trabajo con espacios en blanco disponibles para ser llenados, exige al estudiante realizar un trabajo en clase y consignar sus observaciones.

Para el maestro resulta evidente la construcción conceptual de los estudiantes en la elaboración de las ideas que van consignando en el módulo.

\section{En cuanto a la orientación del trabajo por medio de preguntas}




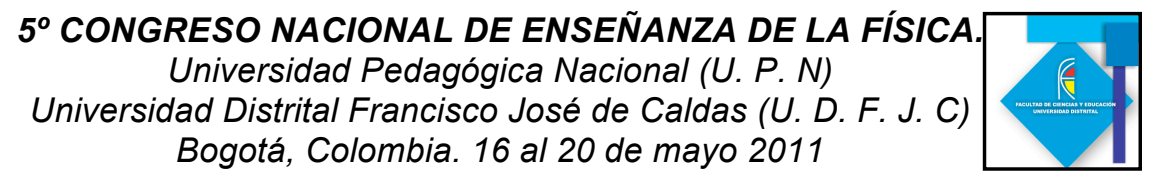

El uso de preguntas permite orientar la línea de pensamiento que lleva el estudiante, pero deben usarse en la medida justa. No se debe atacar al estudiante con muchas preguntas porque se siente perdido y desiste, así como tampoco deben dejarse las preguntas en el aire.

Plantear preguntas orientadoras en el momento preciso de la clase, exige al maestro conocer perfectamente el plan de clase y la temática de estudio, de forma tal que puede saltar libremente de una etapa a otra haciendo uso de las preguntas y no hay riesgo de realizar una pregunta cuya respuesta no la conozca el maestro.

Algunos estudiantes en las últimas unidades ya no formulaban las preguntas al maestro, sino que se las formulaban a ellos mismos y buscaban orientarse con otras preguntas; sin embargo al llegar a una conclusión, consultaban su veracidad con el maestro.

\section{En cuanto a la estrategia de aula}

El uso de experimentos demostrativos orientados por preguntas y un módulo de trabajo, permitió una construcción conceptual de los estudiantes en torno a las ondas sonoras, estudiándolas desde una mirada netamente fenomenológica y orientada a la experiencia cotidiana.

Para diseñar la estrategia de aula, resulta muy importante conocer la población a la que va dirigida, pues eso permite al maestro diseñar el material que sea significativo para el nivel de construcción conceptual de los estudiantes.

La estrategia de aula fomentó la participación y el trabajo en clase de los estudiantes, que es una de sus principales dificultades durante la clase de física.

\section{Bibliografía}

Bautista, E. (2005). Acerca de la experimentación en la enseñanza de las ciencias. Bogotá: Universidad Pedagógica Nacional.

Bellot, D., Cantero, A., Losada, J. A., \& Menéndez, A. L. (2003). El experimento demostrativo en las clases de ciencias naturales de secundaria básica: una variante metodológica para su desarrollo y perfeccionamiento

Delors, J. (1999). La educación encierra un tesoro. UNESCO

Díaz, F., \& Hernández, G. (2003). Estrategias docentes para un aprendizaje significativo (una interpretación constructivista) (segunda ed.). México: Mc Graw Hill

Feynman, R. P., \& Leighton, R. B. (1963). Física: Mecánica, radiación y calor (Vol. I). California: California institute of technology

Giancoli, D. C. (2006). FÍSICA. Principios con aplicaciones. Sexta edición. México: Pearson educación.

Hewitt, P. G. (2004). Conceptos de física. México: Limusa 


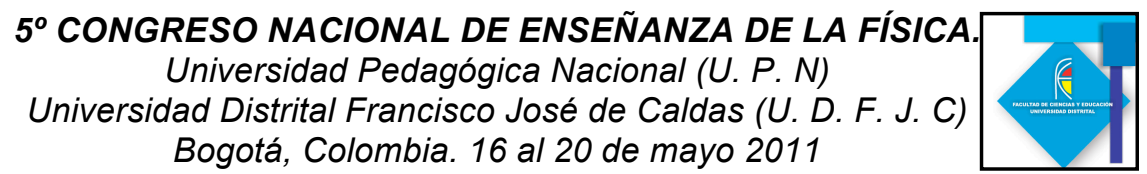

López, M. J. (2009). Construcción de conocimiento. Bogotá: Escuela superior de administración pública ESAP

Ministerio de Educación Nacional [MEN]. (1996). Estándares básicos en competencias, Bogotá.

Ministerio de Educación Nacional [MEN] (1998). Lineamientos curriculares Área de ciencias naturales, Bogotá.

Montes, C. M. (2010). Lineamientos para la construcción de documentos orientadores de prácticas experimentales. Bogotá, Universidad Pedagógica Nacional.

Novak, J. (1988). El constructivismo humano: hacia la unidad en la elaboración de significados psicológicos y epistemológicos.

Resnick, R., Halliday, D., \& Krane, K. S. (1997). Física. México: continental, S. A. de C.V

Vargas, J. E., \& Ramírez, L. F. (2005). Hacia una construcción y comprensión de la acústica. Bogotá: Universidad Pedagógica Nacional. 